\title{
Oleanolic acid liposomes with polyethylene glycol modification: promising antitumor drug delivery
}

This article was published in the following Dove Press journal:

International Journal of Nanomedicine

5 July 2012

Number of times this article has been viewed

\section{Dawei Gao \\ Shengnan Tang \\ Qi Tong}

Applied Chemical Key Laboratory of Hebei Province, College of Environmental and Chemical Engineering, Yanshan University, Qinhuangdao, China

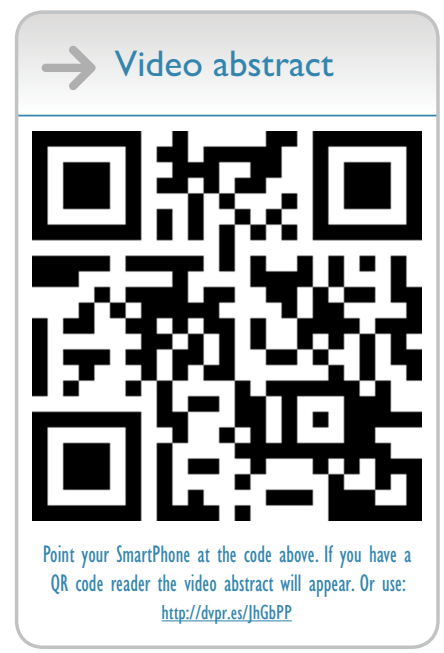

Correspondence: Dawei Gao Applied Chemical Key Laboratory of Hebei Province, College of Environmental and Chemical Engineering, Yanshan University, 438 Hebei Street, Qinhuangdao 066004, China

Tel +86 I393033 8376

Fax +86 $335806 I 569$

Email dwgao@ysu.edu.cn
Background: Oleanolic acid is a pentacyclic triterpene present in many fruits and vegetables, and has received much attention on account of its biological properties. However, its poor solubility and low bioavailability limit its use. The objective of this study was to encapsulate oleanolic acid into nanoliposomes using the modified ethanol injection method.

Methods: The liposomes contain a hydrophobic oleanolic acid core, an amphiphilic soybean lecithin monolayer, and a protective hydrophilic polyethylene glycol (PEG) coating. During the preparation process, the formulations described were investigated by designing $3^{4}$ orthogonal experiments as well as considering the effects of different physical characteristics. The four factors were the ratios of drug to soybean phosphatidylcholine $(w / w)$, cholesterol $(w / w)$, PEG$2000(\mathrm{w} / \mathrm{w})$, and temperature of phosphate-buffered saline at three different levels. We identified the optimized formulation which showed the most satisfactory lipid stability and particle formation. The morphology of the liposomes obtained was determined by transmission electron microscopy and atomic force microscopy. The existence of PEG in the liposome component was validated by Fourier transform infrared spectrum analysis.

Results: The PEGylated liposomes dispersed individually and had diameters of around $110-200 \mathrm{~nm}$. Encapsulation efficiency was more than $85 \%$, as calculated by high-performance liquid chromatography and Sephadex ${ }^{\circledR}$ gel filtration. Furthermore, when compared with native oleanolic acid, the liposomal formulations showed better stability in vitro. Finally, the cytotoxicity of the oleanolic acid liposomes was evaluated using a microtiter tetrazolium assay.

Conclusion: These results suggest that PEGylated liposomes would serve as a potent delivery vehicle for oleanolic acid in future cancer therapy.

Keywords: oleanolic acid, liposomes, ethanol injection, polyethylene glycol

\section{Introduction}

Oleanolic acid is a naturally occuring triterpenoid, widely distributed in the free or glucoside state in nearly 200 types of plant, including Mile swertia, Ligustrum lucidum, and Vitis vinifera. Oleanolic acid has many important biological actions, including anti-inflammatory, antioxidant, and antidiabetic properties. ${ }^{1-3}$ Recently, this compound has been noted for its antitumor effect. ${ }^{4}$ However, being a poorly water-soluble drug $\left(20^{\circ} \mathrm{C}, 4.61 \mathrm{mg} / \mathrm{L}\right)$, the solubility and dissolution rate of oleanolic acid in the gastrointestinal tract is poor, which limits its absorption and bioavailability. ${ }^{5}$

To avoid these adverse effects and the inconvenience of native oleanolic acid, alternative formulations have been exploited for oral delivery of oleanolic acid. Polymeric microparticles and nanoparticles, ${ }^{6}$ lipid nanoparticles, and liposomes have been investigated, ${ }^{7,8}$ and all of these formulations have improved the solubility of 
oleanolic acid. According to the Ostward-Freundlich and Noyes-Whitney equations, the saturation, solubility, and dissolution rate of a drug can be increased by reducing the particle size to increase the interfacial surface area. ${ }^{9}$ Many approaches have been attempted to produce submicron drug powders. The common way of reducing particle size is to disrupt previously formed larger particles using milling methods. In addition, some new techniques, including high-pressure homogenization, have also been developed. However, these techniques show some disadvantages in practice, such as electrostatic effects and broad particle size distribution. In the past decade, nanoparticulate systems have shown the potential to modify drugs by decreasing their toxicity, sustaining their release, and increasing their efficacy, stability, and solubility. ${ }^{10}$ Several nutraceuticals, including curcumin, coenzyme Q, and thymoquinone, have been packaged into nanoparticles and shown to be useful in "nanochemoprevention" and "nanochemotherapy". ${ }^{11}$ Among the most promising drug delivery systems, liposomes are an attractive option for advantageous drug transport. ${ }^{8}$ They are self-assembled nanoparticles and have been used to encapsulate hydrophobic and hydrophilic drugs. ${ }^{12}$ Liposomes have many advantages, including good biocompatibility, biodegradability, low toxicity, and controlled release of the entrapped drug. ${ }^{13}$ In particular, small unilamellar vesicles enter into cells easily, and are beneficial for drug uptake. Liposomes with a diameter of less than $150 \mathrm{~nm}$ have been reported to be suitable for efficient drug delivery. ${ }^{14,15}$ Generally, small unilamellar vesicles can be produced by sonication, extrusion through membranes, and French press extrusion. ${ }^{16}$

Despite the advantages of liposomes, they have some important drawbacks, including batch-to-batch variation in manufacturing, low drug loading efficiency, and poor stability. ${ }^{17}$ Stability is one of the essential aspects of a liposomal drug, which has been the reason for developing formulations with specific steric stabilization. Therefore, various strategies have been developed, including modification of the surface of nanoparticles with phosphatidylinositol or polyethylene glycol (PEG). ${ }^{18,19}$ The latter, known as PEGylation, is one of the most successful approaches for prolonging nanoparticle circulation time in the blood, which leads to better targeting of the encapsulated drug, whilst exploiting the enhanced permeability and retention effect in tumors. ${ }^{20}$ Recently, PEG-lipid conjugates have been incorporated into lipid bilayers, ${ }^{21-23}$ and it has been demonstrated that PEGmodified liposomes have significantly increased stability. ${ }^{24}$

Lately, nanoscale formulations of oleanolic acid have been reported, and oleanolic acid has been successfully encapsulated into liposomes. Most of the work to date has focused on plain oleanolic acid liposomes, and there has been no report of preparation of PEG-modified liposomes. In our study, oleanolic acid was encapsulated in a nanoscale carrier to improve its bioavailability and take advantage of its small size and surface effects. Good solubility and diffusion permeability of oleanolic acid were obtained, along with a reduction in the effective drug dose and a dramatic increase in drug uptake. A modified ethanol injection method combined with sonication was used to prepare oleanolic acid liposomes modified by PEG-2000. Transmission electron microscopy and atomic force microscopy were used to observe the morphology of the oleanolic acid liposome formulation. The anticancer effects of native oleanolic acid and liposomal oleanolic acid on HeLa cells were demonstrated by MTT assay.

\section{Materials and methods Materials}

Oleanolic acid and soybean phosphatidylcholine were purchased from Shenyang Tianfeng Biological Pharmaceutical Co, Ltd (Shenyang, China). Cholesterol, anhydrous ethanol, surfactant Tween-80, and PEG-2000 were purchased from Tianjin Guangfu Fine Chemical Research Institute (Tianjin, China). Sephadex G-75 ${ }^{\circledR}$ and phosphate-buffered saline were obtained from Sigma Chemical Company (Henan, China). All reagents used were of at least analytical grade.

\section{Preparation of PEGylated liposomal oleanolic acid}

In this investigation, liposomes were prepared using the ethanol injection technique. Natural phospholipids showed high stability and biocompatibility, enabling production of lipid vesicles which were more resistant to oxidation. ${ }^{25}$ Moreover, the cost of liposomal formulations based on natural phospholipids is usually lower than that of preparations containing synthetic phospholipids. Cholesterol was used as one of the membrane materials in the preparation of the liposomes in order to improve lipid packaging and membrane permeability characteristics.

Liposomes were prepared according to the ethanol injection method described by Justo and Moraes. ${ }^{26}$ The method used for production of up to $10 \mathrm{~mL}$ of a liposome dispersion is as follows. In brief, oleanolic acid was dissolved in anhydrous ethanol to produce an ethanolic solution of oleanolic acid at a concentration of $0.5 \mathrm{mg} / \mathrm{mL}$. For lipid injection, $9 \%(\mathrm{w} / \mathrm{v})$ soybean phosphatidylcholine, $1.25 \%(\mathrm{w} / \mathrm{v})$ cholesterol, and $0.1 \%(\mathrm{w} / \mathrm{v})$ oleanolic acid were solubilized in 
$2 \mathrm{~mL}$ of anhydrous ethanol as the lipid phase, which was continuously stirred at $43^{\circ} \mathrm{C}$. Tween- $80(0.2 \%[\mathrm{v} / \mathrm{v}]$ in the aqueous phase) and PEG-2000 were dissolved in $10 \mathrm{~mL}$ of phosphate-buffered saline ( $\mathrm{pH}$ 6.5) in a water bath at $43^{\circ} \mathrm{C}$ as the aqueous phase. When the soybean phosphatidylcholine and cholesterol were completely dissolved in the ethanolic solution of oleanolic acid, the lipid phase was dripped into the aqueous phase under magnetic stirring, using a $4 \mu \mathrm{m}$ diameter stainless steel needle attached to a $1 \mathrm{~mL}$ glass syringe. Residual ethanol in the primary emulsion was removed by a rotary evaporator at $45^{\circ} \mathrm{C}$. The liposomal dispersion obtained was then subjected to probe ultrasonic testing using an LTD JY92-II device (Scientz Biotechnology Co, Zhejiang, China) in an ice-bath with a one-second pulse-on period and a one-second pulse-off period for 7 minutes. The probe ultrasonic frequency used in the experiment was around $100 \mathrm{~W}$. Homogeneous and slightly opalescent suspensions were produced, and non-PEGylated liposomes were prepared using the same method. During the study, the ratios of drug to soybean phosphatidylcholine (w/w), cholesterol (w/w), and PEG-2000 (w/w), along with temperature of the phosphatebuffered saline were considered to be important factors for efficacy of the formulation. Furthermore, orthogonal experiments were designed at three levels (Tables 1 and 2). Of note is that the final concentration of ethanol in phosphatebuffered saline was kept constant at $2.5 \%(\mathrm{v} / \mathrm{v})$. In addition, in order to improve the solubility of the preparations, the lipids suspended in ethanol were heated to 43,48 , and $53^{\circ} \mathrm{C}$ before injection. All the liposome suspensions were kept at $4^{\circ} \mathrm{C}$ until further testing.

\section{Determination of entrapment efficiency and drug content}

\section{Gel chromatography separation}

A gel chromatographic column $(1.0 \mathrm{~cm} \times 25 \mathrm{~cm})$ filled with Sephadex G-75 was used to separate the liposomal nanoparticle systems. A $1 \mathrm{~mL}$ sample of liposomal oleanolic acid suspension was added to the column, followed by elution using double-distilled water. Free drug remained in the gel.

Table I Levels of experimental factors

\begin{tabular}{llll}
\hline $\begin{array}{l}\text { Ratio of } \\
\text { OA to SPC } \\
(w / w)\end{array}$ & $\begin{array}{l}\text { Ratio of } \\
\text { OA to Chol } \\
(w / w)\end{array}$ & $\begin{array}{l}\text { Ratio of OA } \\
\text { to PEG-2000 } \\
(w / w)\end{array}$ & $\begin{array}{l}\text { Temperature } \\
\text { of phosphate- } \\
\text { buffered saline }\left({ }^{\circ} \mathbf{C}\right)\end{array}$ \\
\hline I:I5 & I:I & I:I & 43 \\
I:I8 & $\mathrm{I}: 2$ & $2: 3$ & 48 \\
I:20 & $\mathrm{I}: 3$ & $\mathrm{I}: 2$ & 53 \\
\hline
\end{tabular}

Abbreviations: OA, oleanolic acid; Chol, cholesterol; SPC, soybean phosphatidylcholine; PEG, polyethylene glycol.
Table 2 Orthogonal experiment design and drug entrapment efficiency (\%)

\begin{tabular}{|c|c|c|c|c|c|}
\hline & A & B & C & D & EE (\%) \\
\hline I & I & 1 & I & 1 & 80.1 \\
\hline 2 & I & 2 & 2 & 2 & 94.6 \\
\hline 3 & I & 3 & 3 & 3 & 74.5 \\
\hline 4 & 2 & 1 & 2 & 3 & 65.7 \\
\hline 5 & 2 & 2 & 3 & I & 92.8 \\
\hline 6 & 2 & 3 & 1 & 2 & 81.6 \\
\hline 7 & 3 & I & 3 & 2 & 63.9 \\
\hline 8 & 3 & 2 & I & 3 & 98.2 \\
\hline 9 & 3 & 3 & 2 & I & 75.7 \\
\hline $\mathrm{KI}$ & 249.2 & 209.7 & 259.9 & 248.6 & \\
\hline K2 & 240.1 & 285.6 & 236 & 240.1 & \\
\hline K3 & 237.8 & 231.8 & 231.2 & 238.4 & \\
\hline KI & 83.1 & 69.9 & 86.6 & 82.9 & \\
\hline $\mathrm{K} 2$ & 80.0 & 95.2 & 78.7 & 80.0 & \\
\hline K3 & 79.3 & 77.3 & 77.1 & 79.5 & \\
\hline $\mathrm{R}$ & 3.8 & 25.3 & 9.5 & 3.4 & \\
\hline
\end{tabular}

Notes: A, ratio of OA to SPC (w/w); B, ratio of OA to Chol (w/w); C, ratio of OA to PEG-2000 (w/w); D, temperature of phosphate-buffered saline.

Abbreviations: OA, oleanolic acid; Chol, cholesterol; SPC, soybean phosphatidylcholine; PEG, polyethylene glycol; EE, entrapment efficiency.

Liposomes travelling in the gel were collected from the first $6 \mathrm{~mL}$, and the following $50 \mathrm{~mL}$ elution volume was the free oleanolic acid.

\section{HPLC analysis}

The original liposomes and the eluted liposomes were ruptured using an adequate volume of methanol, and the total drug content as well as drug content in the liposomal dispersion were analyzed respectively on an Agilent 1200 series system (Agilent, Santa Clara, CA). The chromatographic apparatus consisted of an Agilent Zorba $\times 300$ SB-C18 column $(5 \mu \mathrm{m}, 250 \mathrm{~mm} \times 4.6 \mathrm{~mm})$ used at room temperature. The mobile phase, consisting of methanol and $0.1 \%$ acetic acid $(88: 12)(\mathrm{v} / \mathrm{v})$, was maintained at a flow rate of $1.0 \mathrm{~mL} /$ minute. The ultraviolet detector wavelength was $215 \mathrm{~nm}$ and the injection volume was $20 \mu \mathrm{L}$. The peak area of response to the concentration of oleanolic acid was linear over the range $10-120 \mu \mathrm{g} / \mathrm{mL}(\mathrm{r}=0.9999)$. Drug entrapment efficiency (EE) and drug loading (DL) were calculated using equations (1) and (2)

$$
\begin{aligned}
& \mathrm{EE} \%=\mathrm{W}_{\text {entrapped }} / \mathrm{W}_{\text {total }} \times 100 \% \\
& \mathrm{DL} \%=\mathrm{W}_{\text {entrapped }} / \mathrm{W}_{\text {lipids }} \times 100 \%
\end{aligned}
$$

where $\mathrm{W}_{\text {entrapped }}$ is the analyzed weight of the amount of drug entrapped in the liposomes, $\mathrm{W}_{\text {total }}$ is the analyzed weight of drug in the liposomal dispersion, and $\mathrm{W}_{\text {lipids }}$ is the total weight of the lipid content. 


\section{Characterization of PEGylated oleanolic acid}

Transmission electron microscopy

Morphological observation of PEGylated liposomal oleanolic acid was done using an transmission electron microscope (JEM-100CX/II, JEOL Ltd, Tokyo, Japan). Samples were diluted with double-distilled water and placed on a copper grid coated with carbon film and air-dried. They were then stained with $2 \%(\mathrm{w} / \mathrm{v})$ tungstophosphoric acid. Finally, the samples were air-dried prior to placing them in the transmission electron microscope at room temperature.

\section{Atomic force microscopy}

Atomic force microscopy was also exploited to investigate the surface morphology of the liposomes in greater detail. Imaging and force measurement were conducted using a Nanoscope IIIa atomic force microscope from Digital Instruments (Bruker AXS Inc, Madison, WI) equipped with an E scanner, having a maximum scan area of $10 \mu \mathrm{m}^{2}$, with scanning performed at room temperature. The scanner was calibrated following the standard procedures recommended by Digital Instruments. A Scan Asyst mode was used to obtain images of the liposomes. Just before analysis, the samples were appropriately diluted in double-distilled water, and $20 \mu \mathrm{L}$ of the dispersion was deposited on mica. The mica was then air-dried on a clean bench. Freshly cleaved mica was mounted onto a stainless steel disc using a sticky tab (Latham, NY) and measurements were performed.

\section{Fourier transform infrared spectrum analysis}

The oleanolic acid liposomes were first freeze-dried to powder, and mannitol and sucrose at a mass ratio of 1:1 were used as lyoprotectants. For preparation, a small quantity of the sample was mixed with dry potassium bromide $(\mathrm{KBr})$, and the mixture was then ground into fine powder using an agate mortar before pressing into a thin $\mathrm{KBr}$ pellet under a hydraulic press at 10,000 psi. Infrared spectra of liposomal oleanolic acid and PEG-2000 samples were obtained in transmission mode using a Fourier transform infrared (FTIR) Per kin-Elmer spectrometer model Spectrum GX from discs containing samples of the liposomes and $\mathrm{KBr}$ in the range of $4000 \mathrm{~cm}^{-1}$ to $400 \mathrm{~cm}^{-1}$. Characteristic peaks were recorded for the different samples.

\section{Stability of PEGylated liposomal oleanolic acid}

Liposomes have many advantages, but also have their limitations. In particular, they tend to adhere to each other and fuse to form larger particles in suspension. In our study, two methods were used to determine and evaluate their stability. First, according to their drug content, PEGylated liposomal oleanolic acid samples were placed in an electric blast-drying oven at 30,40 , and $50^{\circ} \mathrm{C}$. Thereafter, they were kept for 5 , 10 , and 30 days, with their drug contents determined at these respective time points. Entrapment efficiency was evaluated for the PEGylated oleanolic acid liposomes at $20^{\circ} \mathrm{C}$ and $4^{\circ} \mathrm{C}$ on days 1 and 7, at which times leakage rates for the liposomes were also obtained.

\section{In vitro drug release study}

The rate of release of oleanolic acid liposomes in vitro was determined using a dynamic dialysis method in phosphatebuffered saline ( $\mathrm{pH}$ 7.4). Briefly, the dialysis bags were pretreated in the usual way, and $1 \mathrm{~mL}$ of the oleanolic acid liposome solution was then placed into the dialysis bag (molecular weight cutoff 12-14 kDa). The liposome solution was then dialyzed against $150 \mathrm{~mL}$ of phosphate-buffered saline solution at $37^{\circ} \mathrm{C} \pm 0.2^{\circ} \mathrm{C}$ using a rotational speed of $100 \mathrm{rpm}$. At scheduled time intervals, one of the dialysis bags was removed. Meanwhile, the same amount of release medium was added. Finally, the residual oleanolic acid content of the liposomes in the dialysis bags was determined by high-pressure liquid chromatography, as described earlier. Native oleanolic acid and non-PEGylated oleanolic acid liposomes were evaluated as controls. All experiments were performed in triplicate.

\section{In vitro MTT cytotoxicity assay}

The cytotoxic activity of the liposomes was evaluated using the MTT method with HeLa cells. ${ }^{27,28}$ Briefly, HeLa cells were seeded at a density of $5 \times 10^{4} / \mathrm{mL}$ in a 96-well flatbottomed plate and incubated for 24 hours at $37^{\circ} \mathrm{C}$ in a $5 \%$ $\mathrm{CO}_{2}$ atmosphere. Oleanolic acid liposomes were dissolved and diluted in phosphate-buffered saline ( $\mathrm{pH} 7.4$ ), then filtered using a $0.22 \mu \mathrm{m}$ sterile filter and injector. Various drug concentrations of $500,250,125,62.5$, and $31.25 \mu \mathrm{g} / \mathrm{mL}$ were obtained. Non-PEGylated oleanolic acid liposomes dissolved in phosphate-buffered saline at the same dilution were used as a control. Meanwhile, oleanolic acid was dissolved in dimethyl sulfoxide and diluted similarly with phosphatebuffered saline. It is noteworthy that the final concentration of dimethyl sulfoxide in the culture medium was controlled at less than $0.5 \%(\mathrm{v} / \mathrm{v})$. The cells were cultured for 24 hours under the conditions mentioned above. The drugs were added into the appropriate wells, and cell viability was then analyzed using the MTT agent. Briefly, $10 \mu \mathrm{L}$ of MTT solution 
was added to each well, and the cells were incubated for a further 4 hours. Then, $100 \mu \mathrm{L}$ of formazan lysis buffer was added to dissolve any formazan crystals formed. Absorbance was then measured at $595 \mathrm{~nm}$ on a microplate reader. All experiments were performed in triplicate. Absorbance of untreated cells was considered to be $100 \%$ cell viability, and treated cell viability was calculated.

\section{Results \\ Encapsulation efficiency of PEGylated oleanolic acid liposomes}

PEGylated oleanolic acid liposomes prepared using the ethanol injection method were found to be a mildly translucent liposomal suspension. For all experiments using the orthogonal design, entrapment efficiency was 30\%-98\%. Consequently, we chose the optimized formulation to prepare blank and oleanolic acid-loaded liposomes. There were many factors to take into account in the preparation process, including prescription and technology factors. In this study, the ratios of drug to soybean phosphatidylcholine (w/w), cholesterol (w/w), and PEG-2000 (w/w), along with the temperature of the phosphate-buffered saline were identified to be the most influential factors (labeled as A, $\mathrm{B}, \mathrm{C}$, and D in Table 1). Whilst considering the encapsulation efficiency as an important index, the four factors were investigated at three different levels. The $\mathrm{L}_{9}\left(3^{4}\right)$ orthogonal design is shown in Tables 1 and 2. The results of this experiment show that the ranking of the four factors in this experiment was $\mathrm{B}>\mathrm{C}>\mathrm{A}>\mathrm{D}$, the most effective factor being the ratio of drug to cholesterol, with the reactive temperature also having a slight influence. With regard to the individual factors, the appropriate ratio of drug to soybean phosphatidylcholine (w/w) was $1: 15$, the optimum ratio of drug to cholesterol (w/w) was 1:2, and the appropriate ratio of drug to PEG (w/w) was 1:1. Likewise, the optimal reactive temperature was $43^{\circ} \mathrm{C}$. According to the analytical results, the optimal formulation should be $\mathrm{A}_{1} \mathrm{~B}_{2} \mathrm{C}_{1} \mathrm{D}_{1}$.

\section{Drug content}

As shown in Table 3, the oleanolic acid content in the liposomal formulations was relatively high. A content of more than $98 \%$ was clearly observed at the three levels. In particular, the oleanolic acid content at level 3 was 102.37 , which could be explained by the fact that, during the preparation procedure, part of the aqueous medium used to hydrate the oil phase was evaporated, resulting in an increased oleanolic acid concentration in the samples. The results indicate that drug loss in the preparation was not significant.
Table 3 Drug content of three batches

\begin{tabular}{lllll}
\hline $\begin{array}{l}\text { OA/ } \\
\text { SPC }\end{array}$ & $\begin{array}{l}\text { OA/ } \\
\text { Chol }\end{array}$ & $\begin{array}{l}\text { OA/ } \\
\text { PEG-2K }\end{array}$ & Temperature $\left({ }^{\circ} \mathrm{C}\right)$ & DC (\%) \\
\hline I:I5 & I:I & I:I & 43 & $98.75 \pm 0.1 \mathrm{I}$ \\
I:I8 & I:2 & I:I.5 & 48 & $99.72 \pm 0.24$ \\
I:20 & I:3 & I:2 & 53 & $101.7 \pm 0.87$ \\
\hline $\begin{array}{l}\text { Abbreviations: } \\
\text { phosphatidylcholine; PEG, of polyethylene glycol; DC, drug content. }\end{array}$ &
\end{tabular}

\section{Characterization of PEGylated oleanolic acid liposomes}

Morphological examination and particle size measurement of the PEGylated oleanolic acid liposomes were performed using a transmission electron microscope. Figure 1 shows the structures of PEGylated oleanolic acid liposomes with and without surfactant Tween-80. Mean particle size was approximately $100 \mathrm{~nm}$ on representative transmission electron microscopic images. Spherical particles with an obvious double-layer structure were observed. Figure 1B shows that the liposomes with Tween-80 had a characteristic liposomal dispersion, with few particles adhered to each other.

Atomic force micrographs (Figure 2) demonstrated that the size of the PEGylated liposomal oleanolic acid formulations was below $200 \mathrm{~nm}$. Mean particle diameters without Tween-80 surfactant obtained by atomic force microscopy were around $168 \mathrm{~nm}$ (Figure 2A and B). The atomic force micrographs (Figure 2A and B) also showed that only some of the particles adhered to each other when using soybean phosphatidylcholine, and some even aggregated together in bulk. Some particles seemed to be liposomes, judging from their relatively flat shapes, which may be the result of deformation during atomic force microscopy. The mean diameter of the oleanolic acid liposomes prepared from the formulation containing Tween-80 was $110 \mathrm{~nm}$ (Figure 2C and 2D), and it appeared that particle aggregation occurred infrequently
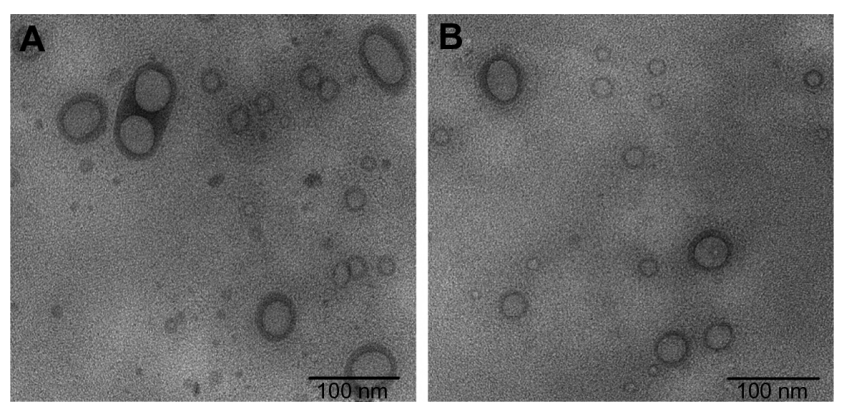

Figure I Transmission electronic microscopic images of PEGylated OA liposomes. (A) Formulation without Tween-80. (B) Tween-80 0.2\% (v/v) added to preparation.

Abbreviations: OA, oleanolic acid; PEG, polyethylene glycol. 

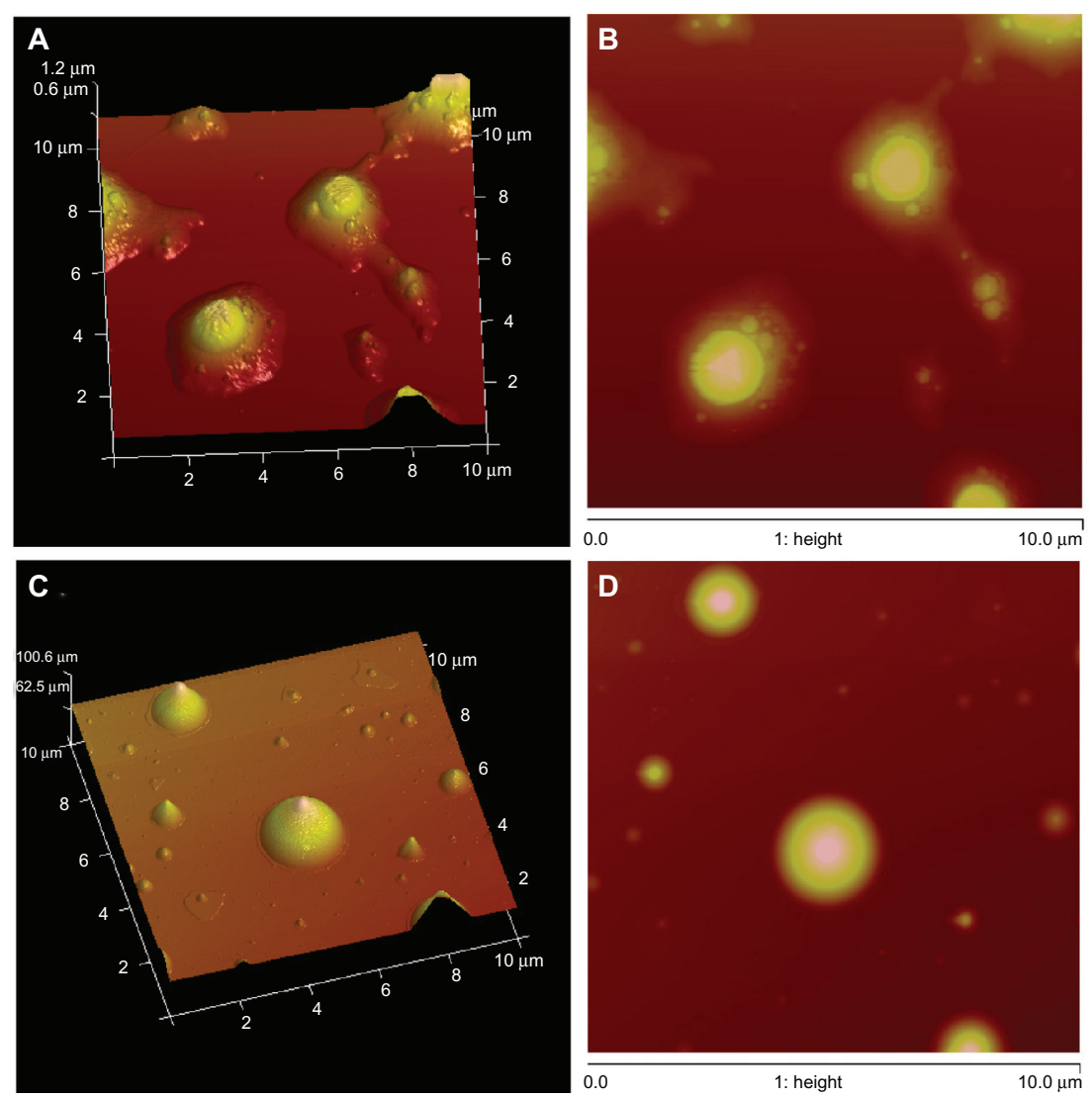

Figure 2 Scan Asyst mode atomic force microscopic images of PEGylated OA liposomes. (A and B) Without Tween-80 and (C and D) with $0.2 \%(\mathrm{v} / \mathrm{v}) \mathrm{Tween}-80$ as a surfactant. Abbreviations: OA, oleanolic acid; PEG, polyethylene glycol.

among PEGylated oleanolic acid liposomes containing Tween-80.

To confirm attachment of PEG to the oleanolic acid liposomes, the liposome formulations were investigated by FTIR spectrometry (Figure 3). Broad strong bands between

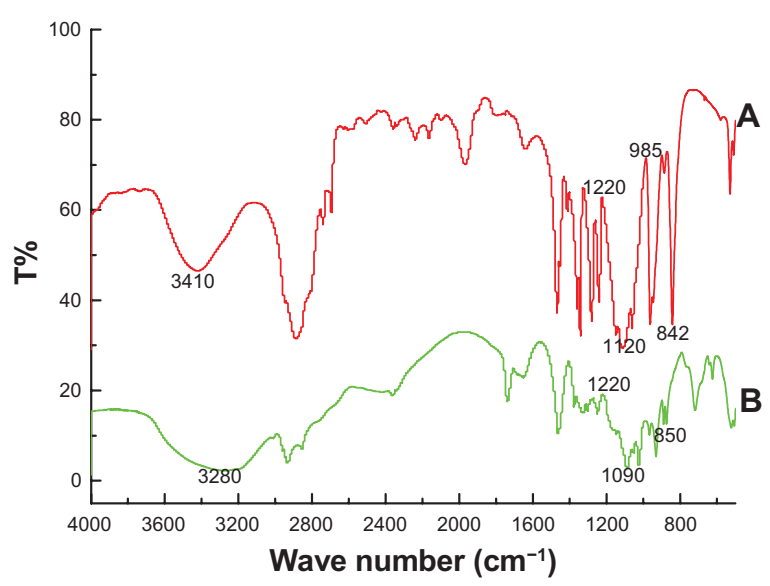

Figure 3 Fourier transform infrared spectra of (A) pure PEG-2000 and (B) PEGylated OA liposomes.

Note: $2 \%(w / w)$ of sample was mixed with dry potassium bromide, compressed into $\mathrm{KBr}$ disc and then scanned in the range of $4000 \mathrm{~cm}^{-1}$ to $400 \mathrm{~cm}^{-1}$.

Abbreviations: OA, oleanolic acid; PEG, polyethylene glycol.
$3340 \mathrm{~cm}^{-1}$ and $3424 \mathrm{~cm}^{-1}$ were seen in the FTIR spectrum of PEG, which corresponds to the stretching vibrations of the intramolecular hydrogen bonds $\left(v_{\mathrm{O}-\mathrm{H}}\right)$ of PEG. Moreover, the typical absorption band at approximately $1120 \mathrm{~cm}^{-1}$ is attributable to the characteristic $\mathrm{C}-\mathrm{O}-\mathrm{C}$ stretching vibration of the repeated $-\mathrm{OCH}_{2} \mathrm{CH}_{2}-$ units of the PEG backbone (Figure 3A). A similar result has been reported by Kassim et al ${ }^{29}$ and Petrova et al. ${ }^{30}$ Pure PEG also showed distinct peaks at about 1220,1120, and $842 \mathrm{~cm}^{-1}$. Characteristic peaks of PEG were also evident in the PEGcoated liposomes, suggesting that PEG was successfully coated onto the liposomes.

\section{Stability of PEGylated oleanolic acid liposomes}

Stability of oleanolic acid liposomes is essential from the nanomedicine point of view. When liposomes are kept for a long time, some leakage of the encapsulated drug can occur. The stability of our PEGylated oleanolic acid liposomes was investigated in two ways. The results obtained using the first method are shown in Table 4. Our experience was that temperature was an important factor in the rate of leakage. 
Table 4 Drug contents of PEGylated OA liposomes in different temperatures after several days

\begin{tabular}{lllll}
\hline Temperature $\left({ }^{\circ} \mathbf{C}\right)$ & $\mathbf{0}$ & $\mathbf{5}$ & $\mathbf{1 0}$ & $\mathbf{3 0}$ \\
\hline 30 & 100 & $99.0 \pm 0.1$ & $98.2 \pm 0.5$ & $97.1 \pm 0.3$ \\
40 & 100 & $98.1 \pm 0.2$ & $97.2 \pm 0.3$ & $94.3 \pm 0.2$ \\
50 & 100 & $95.4 \pm 0.1$ & $94.8 \pm 0.2$ & $90.8 \pm 0.4$ \\
\hline
\end{tabular}

Abbreviations: OA, oleanolic acid; PEG, polyethylene glycol.

The encapsulation efficiency of PEGylated oleanolic acid liposomes decreased as the temperature increased, and after 10 days, the encapsulation efficiency had decreased from $98.2 \%$ to $94.8 \%$. Furthermore, when the liposomes were kept for $5-30$ days at $50^{\circ} \mathrm{C}$, their drug content showed a marked decrease.

The second method was based on the encapsulation efficiency of the nanoparticles. Their physicochemical stability is shown in Table 5. After 14 days, the encapsulation efficiency of non-PEGylated oleanolic acid liposomes had decreased to $70.2 \%$ at room temperature $\left(20^{\circ} \mathrm{C}\right)$. However, the encapsulation efficiency of PEGylated oleanolic acid liposomes had decreased to $76.8 \%$. Oxidation and hydrolysis of the lipids resulted in decomposition and aggregation of the liposomes, which also induced drug leakage. PEGylated oleanolic acid liposomes were stable at low temperature, although their encapsulation efficiency decreased slightly at $4^{\circ} \mathrm{C}$, but their stability was significantly improved.

\section{In vitro drug release study}

The liposomal formulations as well as the native oleanolic acid showed faster release initially and slow release thereafter (Figure 4). Moreover, it was observed that both PEGylated and non-PEGylated oleanolic acid liposomes had a slower release profile than pure oleanolic acid. From 2 to 10 hours, the leakage rate of pure oleanolic acid was markedly higher than for PEGylated oleanolic acid liposomes, and the nonPEGylated formulations showed an intermediate leakage rate. After 10 hours, the leakage rate of PEGylated oleanolic acid liposomes increased significantly, whereas that of pure

Table 5 Entrapment efficiency of PEGylated and non-PEGylated OA liposomes in different conditions

\begin{tabular}{|c|c|c|c|}
\hline Time (d) & I & 7 & 14 \\
\hline \multicolumn{4}{|c|}{ PEGylated OA liposomes } \\
\hline $20^{\circ} \mathrm{C}$ & $96.5 \pm 1.1$ & $86.7 \pm 2.4$ & $76.8 \pm 2.6$ \\
\hline $4^{\circ} \mathrm{C}$ & $97.8 \pm 1.3$ & $91.2 \pm 2.2$ & $85.6 \pm 1.6$ \\
\hline \multicolumn{4}{|c|}{ Non-PEGylated OA liposomes } \\
\hline $20^{\circ} \mathrm{C}$ & $94.3 \pm 1.5$ & $84.1 \pm 1.3$ & $70.2 \pm 2.3$ \\
\hline $4^{\circ} \mathrm{C}$ & $95.6 \pm 1.4$ & $87.6 \pm 1.5$ & $78.6 \pm 2.1$ \\
\hline
\end{tabular}

Abbreviations: OA, oleanolic acid; PEG, polyethylene glycol.

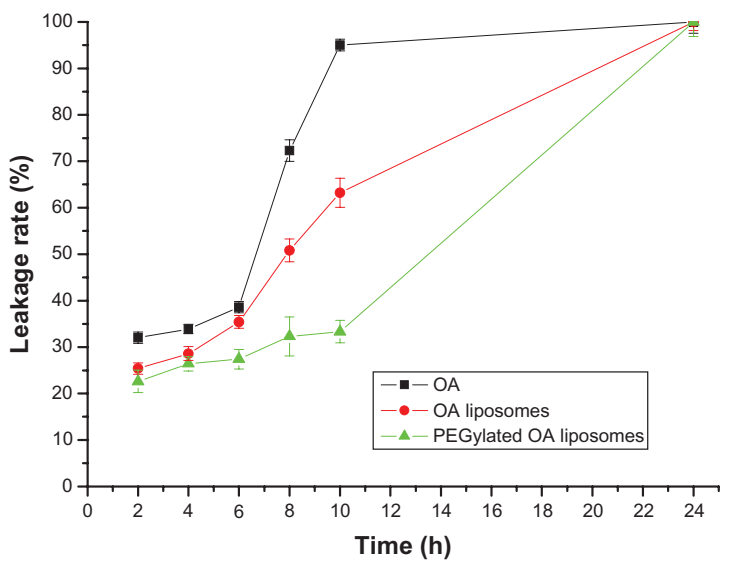

Figure 4 In vitro release of OA, OA liposomes, and PEGylated OA liposomes in phosphate-buffered saline $(0.1 \mathrm{M}, \mathrm{pH} 7.4)$ at $37^{\circ} \mathrm{C}$

Note: Each value represents the mean \pm standard error from three independent experiments.

Abbreviations: OA, oleanolic acid; PEG, polyethylene glycol.

oleanolic acid remained fairly steady. However, leakage from PEGylated and non-PEGylated preparations was consistently lower than that from pure oleanolic acid. These results indicate that oleanolic acid is released more slowly from the PEGylated liposomal formulation than from the pure oleanolic acid formulation, which could reduce drug toxicity.

\section{MTT assay of antitumor activity}

The in vitro antitumor effect of pure oleanolic acid and that of PEGylated and non-PEGylated oleanolic acid liposomes were studied by MTT assay (Figure 5), using oleanolic acid as a model antitumor drug. With regard to viability, the HeLa cell retained different sensitivity to native oleanolic acid and the different kinds of oleanolic acid liposomes. With pure oleanolic acid, the cell viability was higher than

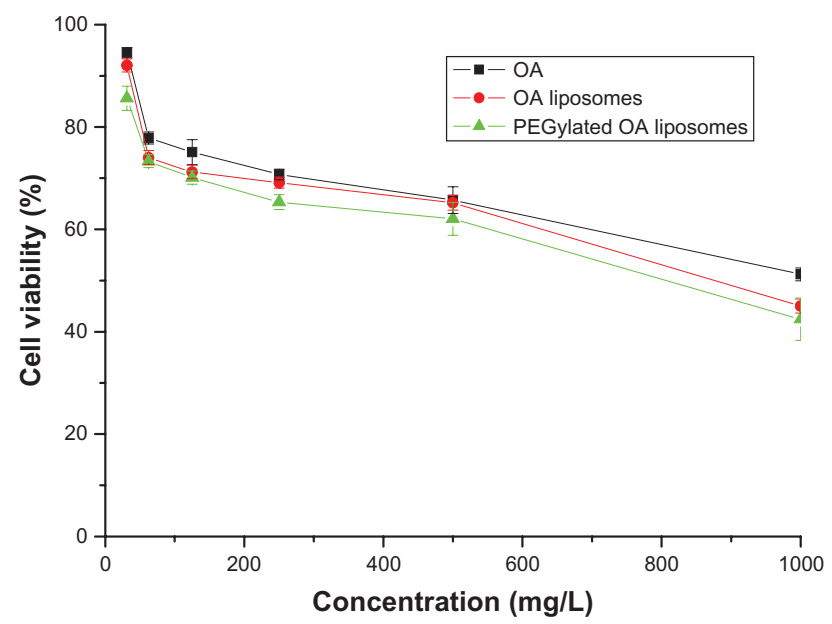

Figure 5 Effect of drug concentration on the inhibition rate of OA, OA liposomes and PEGylated OA liposomes.

Note: Each value represents the mean \pm S.E.M from 3 independent experiments. 
for oleanolic acid-loaded liposomes, and had somewhat low antitumor activity. However, this activity increased significantly when cells were treated with oleanolic acid liposomes and PEGylated formulations. Cell numbers were reduced in a concentration-dependent manner. Moreover, it was demonstrated that antitumor activity was enhanced when oleanolic acid was prepared as a nanoscale medicine. In particular, surface-modified liposomes showed better inhibition of HeLa cells.

\section{Discussion}

Liposomes have been considered to be promising and valuable antitumor drug carriers due to their many advantages. Controlled drug delivery systems using liposomes can improve the biodistribution of existing anticancer drugs, and prolong drug accumulation in both tumor tissue and in the blood stream. The design of liposomes is important because their hydrophobic core should be capable of carrying highly insoluble anticancer drugs with high loading capacity (most chemotherapeutic drugs are hydrophobic). However, a surface-modified hydrophilic moiety provides steric protection and reduces macrophage uptake in vivo. One of the most widely used methods for enhancing the circulation properties of liposomes is incorporation of PEG within the surface membrane of liposomes. ${ }^{19,31,32}$ The possible mechanism is that a hydrated PEG shell disturbs the interaction of liposomes with serum proteins, and significantly reduces opsonization and uptake by macrophages. ${ }^{33}$

The water solubility and bioavailability of oleanolic acid is very low. ${ }^{34}$ Presently, various new oleanolic acid formulations are being developed to overcome the problem of low oral bioavailability. ${ }^{35}$ Oleanolic acid drop pills have been successfully obtained by Tang et al, through mixing homogeneously with PEG of different molecular weights. ${ }^{36}$ However, there have been a few reports of exploiting oleanolic acid liposomes modified by PEG. Consequently, our hypothesis was that a hydrophilic polymeric coating (ie, PEG) could improve the longevity of oleanolic acid in the circulation and have slow-release properties due to stability of the drug-loaded liposomes.

In our study, drug content was determined after the liposomes were fabricated. The results provide some groundwork in the examination of entrapment efficiency. Morphological analysis of the formulated liposomes by transmission electron microscopy provided further evidence of successful incorporation of PEG into nanoparticles. Atomic force microscopy showed that the particle size was larger than that determined by transmission electron microscopy. There were two possible explanations for this difference. One reason is that the structure of the liposomes could be changed when atomic force microscopy was performed. In the process of observing the atomic force microscopic images of the formulations, the force of the probe was not always uniform; in addition, the dried liposome film was very soft. Tapping of the probe resulted in deformation of some liposomes and a larger particle size. Second, the mica plate used to support the samples was not completely flat, which might have induced a morphological change in the liposomes. The incorporation of PEG improves biocompatibility, and decreases the positive surface charge significantly, as reported by Quellec et al. ${ }^{37}$ It has been considered generally that PEGylation forms a fixed aqueous layer on the surface of nanoparticles via interaction between PEG and water molecules, leading to reduced amounts of associated serum opsonins on the surface, thereby prolonging the circulation time of the nanoparticles. ${ }^{38-41}$ Because the Tween- 80 used as a surfactant has a polyoxyethylene chain in its structure, molecules of Tween- 80 located at the interface between the water and oil phase of the emulsion may have a similar function to that of PEG molecules. However, the detailed mechanisms behind these observations for a PEG emulsion, including the conformational and functional properties of Tween- 80 on the surface of the emulsion, remain to be elucidated and will be the subject of our future studies.

Aggregation of liposomes may lead to leakage of inclusions. ${ }^{42,43}$ Therefore, stability is an essential problem for lipid vesicles. ${ }^{44}$ After preliminary studies to find the best formulation for PEG liposomes, an in vitro release study was carried out to evaluate the rate of leakage from the preparations. The results indicate that PEG-modified liposomes had slow release and were stable compared with pure oleanolic acid. The configuration of PEG molecules on the surface of the liposome was important for steric repulsion in vitro because it determined the shielding efficiency. Moreover, PEGylated formulations showed advantages on cytotoxicity assay. The viability of cells treated with oleanolic acid liposomes performed better than oleanolic acid alone, but the pattern approximately resembled that of pure oleanolic acid.

Some earlier studies have been done on oleanolic acid liposomes and PEG-coated liposomes. ${ }^{45,46}$ However, there has not been any detailed investigation of PEGylated oleanolic acid liposomes, which have an important role in evading uptake by macrophages. ${ }^{47,48}$ It was found that high molecular weight PEG (2000 Da and higher) covalently attached to the lipid head group undergoes steric exclusion from the surface of the liposome. By dehydrating the lipid bilayer, high 
molecular weight PEG plays a significant role in the thermodynamic stability of liposomes. ${ }^{49-51}$ Also, hydrophilic PEG chains on the surface of the bilayers can adsorb more water molecules than those entrapped in the core. ${ }^{52,53}$ Thereby, the addition of $\mathrm{PEG}$ could enhance the encapsulating percentage and loading efficiency. PEG-2000 has been selected by many research groups as a surface-modifying polymer to develop novel liposome formulations. ${ }^{54,55}$ Liposomes modified using PEG-2000 showed feasibility and stable potential for loading drugs. Furthermore, it was demonstrated that introduction of PEG-2000 introduced concentricity of size distribution. In addition to protecting the vesicles against peritoneal macrophages, the presence of PEG-2000 reduces repulsion between the negative surfaces of the vesicles and the peritoneal mesothelium. ${ }^{56}$

In our study, PEG-2000 was used to modify conventional liposomes. Formulations with steric stability were successfully obtained. We have confirmed the appropriate concentration of PEG to maximize its antitumor potential as well as optimizing the configuration of formulations. Therefore, we propose to have a mass ratio of oleanolic acid to PEG of 1:1 in our fabricated liposomes, which may be used as a very effective drug delivery system for antitumor drugs. The increased cytotoxicity of PEG-modified oleanolic acid liposomes may open a new pathway for the targeting of anticancer drugs, but there is still a pressing need for further in vivo studies to realize their full potential.

\section{Acknowledgments}

This work was financially supported by a research grant from the Chinese Ministry of Education Doctor Degree (20101333120011), grants from the Hebei Province Natural Science Fund (C2011203137, 11965152D), and a Chinese postdoctoral grant (480013).

\section{Disclosure}

The authors report no conflicts of interest in this work.

\section{References}

1. Tsuruga T, Chun YT, Ebizuka Y, Sankawa U. Biologically active constituents of Melaleuca leucadendron: inhibitors of induced histamine release from rat mast cells. Chem Pharm Bull. 1991;39:3276-3278.

2. Balanehru S, Nagarajan B. Protective effect of oleanolic acid and ursolic acid against lipid peroxidation. Biochem Int. 1991;24:981-990.

3. Zhang YN, Zhang W, Hong D, et al. Oleanolic acid and its derivatives: new inhibitor of protein tyrosine phosphatase 1B wth cellular activities. Bioorg Med Chem. 2008;16:8697-8705.

4. Shibata S. Chemistry and cancer preventing activities of ginseng saponins and some related triterpenoid compounds. J Korean Med Sci. 2001; 16 Suppl:S28-S37.

5. Liu J. Pharmacology of oleanolic acid and ursolic acid. JEthnopharmacol. 1995;49:57-68.
6. Bilensoy E, Gurkaynak O, Dogan AL, Hincal AA. Safety and efficacy of amphiphilic beta-cyclodextrin nanoparticles for paclitaxel delivery. Int J Pharm. 2008;347:163-170.

7. Arica Yegin B, Benoit JP, Lamprecht A. Paclitaxel-loaded lipid nanoparticles prepared by solvent injection or ultrasound emulsification. Drug Dev Ind Pharm. 2006;32:1089-1094.

8. Torchilin VP. Recent advances with liposomes as pharmaceutical carriers. Nat Rev Drug Discov. 2005;4:145-160.

9. Mosharraf M, Nystrom C. The effect of particle size and shape on the surface specific dissolution rate of microsized practically insoluble drugs. Int J Pharm. 1995;122:35-47.

10. Elaine MM, Gary GL. Drug nanoparticles: formulating poorly watersoluble compounds. Toxicol Pathol. 2008;36:43-48.

11. Nair HB, Sung B, Yadav VR, Kannappan R, Chaturvedi MM, Aggarwal BB. Delivery of anti-inflammatory nutraceuticals by nanoparticles for the prevention and treatment of cancer. Biochem Pharmacol. 2010;80:1833-1843.

12. Naik S, Patel D, Chuttani K, Mishra AK, Misra A. In vitro mechanistic study of cell death and in vivo performance evaluation of RGD grafted PEGylated docetaxel liposomes in breast cancer. Nanomedicine. November 22, 2011. doi:10.1016/j.nano.2011.11.008

13. Papahadjopoulos D, Allen TM, Gabizon A, et al. Sterically stabilized liposomes -improvements in pharmacokinetics and antitumor therapeutic efficacy. Proc Natl Acad Sci U S A. 1991;88:11460-11464.

14. Drummond DC, Meyer O, Hong K, Kirpotin DB, Papahadjopoulos D. Optimizing liposomes for delivery of chemotherapeutic agents to solid tumors. Pharmacol Rev. 1999;51:691-743.

15. Takeuchi H, Kojima H, Yamamoto H, Kawashima Y. Passive targeting of doxorubicin with polymer coated liposomes in tumor bearing rats. Biol Pharm Bull. 2001;24:795-799.

16. Szoka F, Papahadjopoulos D. Comparative properties and methods of preparation of lipid vesicles (liposomes). Апnu Rev Biophys Bioeng. 1980;9:467-508.

17. Mishra B, Patel BB, Tiwari S. Colloidal nanocarriers: a review on formulation technology, types and applications toward targeted drug delivery. Nanomedicine. 2010;6:9-24.

18. Allen TM, Hansen C. Pharmacokinetics of stealth versus conventional liposomes: effect of dose. Biochim Biophys Acta. 1991;1068: 133-141.

19. Allen TM, Hansen C, Martin F, Redemann C, Yau-Young A. Liposomes containing synthetic lipid derivatives of poly(ethylene glycol) show prolonged circulation half-lives in vivo. Biochim Biophys Acta. 1991;1066:29-36.

20. Gabizon A, Shmeeda H, Barenholz Y. Pharmacokinetics of pegylated liposomal doxorubicin: review of animal and human studies. Clin Pharmacokinet. 2003;42:419-436.

21. Bista K, Bruch RF, Covington AM, Sorger A, Gerstmann T, Otto A. Investigations of thermotropic phase behavior of newly developed synthetic PEGylated lipids using Raman spectro-microscopy. Biopolymers. 2008;89:1012-1020.

22. Sadzuka Y, Kishi K, Hirota S, Sonobe T. Effect of polyethyleneglycol (PEG) chain on cell uptake of PEG-modified liposomes. J Liposome Res. 2003;13:157-172.

23. Shimada K, Matsuo S, Sadzuka Y, et al. Determination of incorporated amounts of poly(ethylene glycol)-derivatized lipids in liposomes for the physicochemical characterization of stealth liposomes. Int J Pharmacol. 2000;203:255-263.

24. Ishida T, Harashima H, Kiwada H. Liposome clearance. Biosci Rep. 2002;22:197-224.

25. Justo OR, Moraes AM. Analysis of process parameters on the characteristics of liposomes prepared by ethanol injection with a view to process scale-up: effect of temperature and batch volume. Chem Eng Res Des. 2011;89:785-792. (Chemical engineering research and design. ISSN:0263-8762).

26. Justo OR, Moraes AM. Kanamycin incorporation in lipid vesicles prepared by ethanol injection designed for tuberculosis treatment. J Pharm Pharmacol. 2005;57:23-30. 
27. Kroll A, Pillukat MH, Hahn D, Schnekenburger J. Current in vitro methods in nanoparticle risk assessment: limitations and challenges. Eur J Pharm Biopharm. 2009;72:370-377.

28. Plumb JA. Cell sensitivity assays: the MTT assay. Methods Mol Med. 1999;28:25-30.

29. Kassim A, Ekarmul Mahmud HNM, Yee LM, Hanipah N. Electrochemical preparation and characterization of polypyrrolepolyethylene glycol conducting polymer composite films. Pac J Sci Technol. 2006;7:103-107. (pdf)

30. Petrova S, Miloshev S, Mateva R, Iliev I. Synthesis of amphiphilic PEG-PCL-PEG triblock copolymers. J Univ Chem Technol Metall. 2008;43:199-204. (pdf)

31. Blime G, Cevc G. Liposomes for the sustained drug release in vivo. Biochim Biophys Acta. 1990;1029:91-97.

32. Klibanov AL, Maruyama K, Torchilin VP, Huang L. Amphipathic poly(ethylene glycol) effectively prolong the circulation time of liposomes. FEBS Lett. 1990;268:235-237.

33. Furumoto K, Yokoe J, Ogawara K, et al. Effect of coupling of albumin onto surface of PEG liposome on its in vivo disposition. Int J Pharm. 2007;329:110-116.

34. Chen YJ, Yang XL, Zhao XL, et al. Preparation of oleanolic acid nanosuspension. J Pharm Pharmacol. 2006;41:924-927.

35. Yang R, Su LQ, Huang X. Preparation and quality evaluation of oleanolic acid self-microemulsion. Food Drug. 2008;10:40-42. Chinese literature.

36. Tang F, Li HD. Dispersion of oleanolic acid drop pills and its evaluation in vitro. Chin Pharm J. 2008;43:1167-1169. Chinese literature.

37. Quellec P, Gref R, Perrin L, et al. Protein encapsulation within polyethylene glycol-coated nanospheres. I. Physicochemical characterization. J Biomed Mater Res. 1998;42:45-54.

38. Shimada K, Miyagishima A, Sadzuka Y, et al. Determination of the thickness of the fixed aqueous layer around polyethyleneglycol-coated liposomes. J Drug Target. 1995;3:283-289.

39. Sadzuka Y, Nakade A, Hirama R, et al. Effect of mixed polyethyleneglycol modification on fixed aqueous layer thickness and antitumor activity of doxorubicin containing liposome. Int J Pharm. 2002;238:171-180

40. Shehata T, Ogawara K, Higaki K, Kimura T. Prolongation of residence time of liposome by surface-modification with mixture of hydrophilic polymers. Int J Pharm. 2008;359:272-279.

41. Yokoe J, Sakuragi S, Yamamoto K, et al. Albumin-conjugated PEG liposome enhances tumor distribution of liposomal doxorubicin in rats. Int J Pharm. 2008;353:28-34.

42. Matteucci ML, Thrall DE. The role of liposomes in drug delivery and diagnostic imaging: a review. Vet Radiol Ultrasound. 2000;41: $100-107$.
43. Pedroso de Lima MC, Neves S, Filipe A, Duzgunes N, Simoes S. Cationic liposomes for gene delivery: from biophysics to biological applications. Curr Med Chem. 2003;10:1221-1231.

44. Bakker Woudenberg IA, Storm G, Woodle MC. Liposomes in the treatment of infections. J Drug Target. 1994;2:363-371.

45. Sheng Y, Liu C, Yuan Y, et al. Long-circulating polymeric nanoparticles bearing a combinatorial coating of PEG and water-soluble chitosan. Biomaterials. 2009;30:2340-2348.

46. Zahr AS, Davis CA, Pishko MV. Macrophage uptake of core-shell nanoparticles surface modified with poly(ethylene glycol). Langmuir. 2006;22:8178-8185.

47. Gbadamosi JK, Hunter AC, Moghimi SM. PEGylation of microspheres generates a heterogeneous population of particles with differential surface characteristics and biological performance. FEBS Lett. 2002;532:338-344.

48. Papisov MI. Theoretical considerations of RES-avoiding liposomes: molecular mechanics and chemistry of liposome interactions. Adv Drug Deliv Rev. 1998;32:119-138.

49. Priev A, Samuni A, Tirosh O, Barenholz Y. The role of hydration in stabilization of liposomes: resistance to oxidative damage of PEGgrafted liposomes. J Target Drug. 1998;6:147-167.

50. Tirosh O, BarenholzY, Katzhendler J, Priev A. Hydration of polyethylene glycol-grafted liposomes. J Biophys. 1998;74:1371-1379.

51. Zalipsky S, Harris MJ. Chemistry and biological applications of poly(ethylene glycol). Presented at ACS Symposium Series 680, April 13-17, 1997. San Francisco, CA.

52. Zucker D, Andriyanov AV, Steiner A, Raviv U, Barenholz Y. Characterization of PEGylated nanoliposomes co-remotely loaded with topotecan and vincristine: relating structure and pharmacokinetics to therapeutic efficacy. J Control Release. October 12, 2011. doi:10.1016/j.jconrel.2011.10.003

53. Kaminskas LM, McLeod VM, Kelly BD, et al. A comparison of changes to doxorubicin pharmacokinetics, antitumor activity, and toxicity mediated by PEGylated dendrimer. Nanomedicine. 2012;8:103-111.

54. Brusa P, Arpicco S, Stella B, Dosio F, Cattel L, Immordino ML. Preparation, characterization, cytotoxicity and pharmacokinetics of liposomes containing docetaxel. J Control Release. 2003;91: 417-429.

55. Naik S, Patel D, Surti N, Misra A. Preparation of PEGylated liposomes of docetaxel using supercritical fluid technology. J Supercrit Fluids. 2010;54:110-119.

56. Dadashzadeh S, Mirahmadi N, Babaei MH, Vali AM. Peritoneal retention of liposomes: effects of lipid composition, PEG coating and liposome charge. J Control Release. 2010;148:177-186.
International Journal of Nanomedicine

\section{Publish your work in this journal}

The International Journal of Nanomedicine is an international, peerreviewed journal focusing on the application of nanotechnology in diagnostics, therapeutics, and drug delivery systems throughout the biomedical field. This journal is indexed on PubMed Central, MedLine, CAS, SciSearch $\AA$, Current Contents $\AA /$ Clinical Medicine,

\section{Dovepress}

Journal Citation Reports/Science Edition, EMBase, Scopus and the Elsevier Bibliographic databases. The manuscript management system is completely online and includes a very quick and fair peer-review system, which is all easy to use. Visit http://www.dovepress.com/ testimonials.php to read real quotes from published authors. 